\section{Satisfação com saúde bucal de idosos brasileiros: um estudo de gênero com modelo hierárquico}

\author{
Oral health satisfaction among Brazilian elderly: \\ a gender study using a hierarchical model
}

\author{
Satisfacción con la salud bucal en adultos mayores \\ brasileños: un estudio de género con el modelo \\ jerárquico
}

\author{
1 Universidade Federal de \\ Minas Gerais, Belo Horizonte, \\ Brasil. \\ Correspondência \\ A. C. V. Campos \\ Universidade Federal de \\ Minas Gerais. \\ Rua Oswaldo Cruz 167, \\ Sete Lagoas, MG 35700-195, \\ Brasil. \\ campos.acv@gmail.com
}

\begin{abstract}
The objectives of this study were to identify gender differences in relation to factors associated with oral health satisfaction among the elderly and to estimate the prevalence of edentulism by gender and age. The study included 2,052 elderly ( $\geq 60$ years) residing in Sete Lagoas, Minas Gerais State, Brazil. Poisson regression was used to estimate prevalence of edentulism. The as sociation between oral health satisfaction and independent variables was estimated using ordinal regression with a hierarchical multidimensional model. Prevalence rates for edentulism in males and females were $63 \%$ and $68.8 \%$, respectively. Most elderly males (63\%) and females (57\%) were satisfied with their oral health. The final adjusted model for both genders consisted of variables related to environmental characteristics, health behaviors, and oral health conditions. The study concludes that oral satisfaction is heavily influenced by oral health status and health behaviors, while the associated factors differ by gender.
\end{abstract}

Oral Health; Aged; Self Concept
Ana Cristina Viana Campos 1 Andréa Maria Duarte Vargas 1 Efigênia Ferreira e Ferreira 1

\section{Resumo}

Os objetivos do presente estudo foram identificar as diferenças de gênero em relação aos fatores associados à satisfação com saúde bucal de idosos e estimar a prevalência de edentulismo por gênero e faixa etária. Foram incluídos 2.052 idosos com 60 anos e mais de idade, residentes em Sete Lagoas, Minas Gerais, Brasil. A regressão de Poisson foi utilizada para estimar a prevalência de edentulismo. A associação entre satisfação bucal e as variáveis independentes foi realizada por meio da regressão ordinal com modelo hierárquico multidimensional. A prevalência de edentulismo nos gêneros masculino e feminino foi de $63 \%$ e 68,8\%, respectivamente. A maioria dos idosos do gênero masculino (63\%) e feminino (57\%) estava satisfeita com a saúde bucal. O modelo final ajustado, para ambos os gêneros, foi formado por variáveis referentes ao ambiente externo, comportamentos de saúde e condições de saúde bucal. Conclui-se que a satisfação com a saúde bucal é influenciada fortemente pelas condições de saúde bucal e por comportamentos de saúde, e que os fatores associados são diferentes para cada gênero.

Saúde Bucal; Idoso; Autoimagem 


\section{Introdução}

O último levantamento epidemiológico realizado pelo Ministério da Saúde em 2010 indica um panorama ainda crítico para os idosos brasileiros, com elevada perda dentária e edentulismo 1 . Um estudo de revisão sistemática sobre a saúde bucal desse grupo citado revelou uma prevalência de edentulismo média de $60,13 \%$ e elevado uso de próteses nas diferentes populações e contextos abordados 2 .

Diante desse quadro, o desenvolvimento de ações sociais de prevenção, diagnóstico e intervenção é cada vez mais necessário ${ }^{3}$. Apesar dos resultados positivos da Política Nacional de Saúde Bucal - Brasil Sorridente - junto à Estratégia Saúde da Família, o uso dos serviços odontológicos é muito baixo entre idosos e pouco se sabe em que medida está relacionado à percepção da necessidade de tratamento odontológico e à autopercepção da saúde bucal 4 .

A obtenção de dados epidemiológicos por meio de índices e indicadores quantitativos é importante, mas leva em conta apenas a visão do profissional 5. Considerando-se que o comportamento dos indivíduos é condicionado por suas percepções e pela importância dada a elas, a avaliação da autopercepção das condições de saúde bucal representa um ganho substancial para o planejamento dos serviços de saúde nessa área 6.

A utilização dos modelos sociológicos de interação entre variáveis subjetivas e objetivas para o estudo de percepção e autoavaliação da saúde bucal da população idosa vem ganhando repercussão internacional 7,8,9. Esses modelos levam em consideração as características individuais, ambientais, sociocomportamentais e culturais, as condições clínicas e também a experiência subjetiva dos indivíduos sobre o seu bem-estar funcional, social e psicológico, refletindo a natureza multidimensional da autoavaliação da saúde bucal 10,11,12.

Concomitantemente, estudos nacionais têm evidenciado que o envelhecimento é diferente para homens e mulheres 13. Em relação à saúde bucal, a maioria dos trabalhos aborda apenas o perfil epidemiológico bucal 14, com amostras não comparáveis entre os gêneros 15,16, e foi realizada com idosos institucionalizados 17.

Portanto, os objetivos do presente estudo foram identificar as diferenças de gênero em relação aos fatores associados à satisfação com saúde bucal de idosos e estimar a prevalência de edentulismo por gênero e faixa etária.

\section{Métodos}

\section{Desenho do estudo e participantes}

Este trabalho é parte de um estudo longitudinal realizado no Município de Sete Lagoas, Minas Gerais, Brasil, com uma população residente de 214.152 habitantes, sendo que 10,16\% têm a idade igual ou superior a 60 anos ${ }^{18}$. O município é dividido em 17 regiões administrativas (criadas para substituir os limites dos 183 bairros existentes) 19 , três áreas urbanas isoladas, um distrito fora da sede e área rural.

O cálculo amostral foi realizado para comparação entre gêneros considerando-se a prevalência de capacidade funcional para atividades instrumentais nos gêneros masculino $(86,6 \%)$ e feminino $(72,9 \%) 20$. O erro estimado foi de até cinco pontos porcentuais, poder de teste de $80 \%$, com intervalos de 95\% de confiança (IC95\%), considerando um efeito de delineamento de dois. Foi utilizado um adicional de $20 \%$ para perdas e recusas. Em seguida, a amostra de cada grupo (homens e mulheres) foi estratificada por faixa etária em relação à população e corrigida pela probabilidade do risco de morrer, resultando em 1.146 idosos.

Os critérios de exclusão adotados foram: indivíduos residentes em instituição de longa permanência no período da coleta de dados, os portadores de deficiências visual e/ou auditiva graves e não corrigidas, aqueles com deficiência cognitiva que impede o entendimento e execução da entrevista e do exame clínico, e os que se recusaram a assinar o Termo de Consentimento Livre e Esclarecido (TCLE).

A técnica de amostragem foi probabilística por conglomerados sob o critério de partilha proporcional ao tamanho em dois estágios: setores censitários e domicílios, que foram sorteados proporcionalmente ao número de domicílios permanentes particulares ocupados por estrato (área urbana, zona rural, distrito).

A coleta de dados foi realizada no domicílio e foram consideradas perdas de entrevista/exame os casos em que o idoso não foi localizado após três tentativas, incluindo o fim de semana. $\mathrm{O}$ projeto foi divulgado junto às autoridades municipais, meios de comunicação e por folder próprio.

O exame epidemiológico foi feito sob iluminação natural, espelho bucal plano e uma espátula de madeira. $\mathrm{O}$ índice CPOD (dentes permanentes cariados, perdidos e restaurados) foi utilizado para estimar o edentulismo, segundo critérios preconizados pela Organização Mundial da Saúde (OMS) 21, mas adaptados. O número de dentes perdidos foi apurado e a variável foi 
dicotomizada em: ausência de todos os dentes e presença de um ou mais dentes.

Foi realizada também uma avaliação clínica sobre uso e necessidade de próteses superior e inferior. Para avaliar uma prótese em usos consideraram-se retenção, estabilidade, e reciprocidade e estética. Os possíveis códigos indicavam o tipo de prótese (parcial removível, parcial fixa, total) e a região (superior, inferior). Neste trabalho, as variáveis foram agrupadas seguindo recomendações da OMS, para medir o "agravamento" do edentulismo 21 .

O questionário estruturado continha 50 perguntas semelhantes às da Pesquisa Nacional por Amostra de Domicílios (PNAD) de 2011, incluindo questões sobre estilo de vida e acesso e utilização de serviços de saúde bucal. Para as variáveis relacionadas à saúde bucal utilizaram-se perguntas selecionadas do SBBrasil 2010. O método teste/ reteste foi empregado para verificar a confiabilidade e o desempenho dos questionários e testes nesta amostra. A fidedignidade foi avaliada pela correlação de Spearman para teste de associação simples entre as variáveis do instrumento, e pela correlação partial rank de Kendall para avaliar a interferência ou não de um fator externo comum a todos os sujeitos. Obtiveram-se coeficientes de correlações superiores a $0,80(p<0,001)$.

O projeto piloto foi conduzido anteriormente à coleta de dados com 107 idosos (10\% da amostra total) em um município vizinho, sendo que as entrevistas foram realizadas em duplicata para verificação das concordâncias inter e intraexaminadores, utilizando-se o coeficiente kappa. Obteve-se $92 \%$ de concordância interexaminadores e $98,5 \%$ para intraexaminadores, seguindo os parâmetros adotados pela OMS 21 .

A coleta de dados foi realizada entre os meses de janeiro e julho de 2012, por uma equipe formada por três examinadores e três anotadores treinados e calibrados. O projeto foi aprovado pelo Comitê de Ética em Pesquisa da Universidade Federal de Minas Gerais (COEP-UFMG), CAAE-0413.0.203.000-11.

\section{Satisfação com a saúde bucal}

Para a variável dependente - satisfação com a saúde bucal ("Com relação aos seus dentes/ boca o(a) senhor(a) estava...”) - as respostas foram agregadas em três categorias: "insatisfeito" (união de muito insatisfeito a insatisfeito), "nem insatisfeito nem satisfeito" e "satisfeito" (união de muito satisfeito a satisfeito), o que permitiu não perder o caráter ordinal entre as categorias da variável.

\section{Modelo teórico conceitual}

O presente estudo utilizou o modelo comportamental em saúde 22 adaptado por Martins et al. 23 para investigar os fatores associados ao grau de satisfação bucal em idosos. As variáveis mais distais são as características demográficas. O modelo comportamental expandido conceitua os comportamentos de saúde, as características do ambiente externo e recursos socioeconômicos como variáveis intermediárias, que por sua vez influenciam os resultados de saúde bucal (avaliada, percebida, satisfação do indivíduo).

As variáveis independentes foram agrupadas em seis níveis hierárquicos distintos entre distal, intermediário e proximal em direção ao desfecho (Figura 1). As variáveis distais, localizadas no primeiro nível de análise, foram denominadas características individuais: faixa etária (60-64 anos, 65-69, 70-74, 75-79 anos, 80 anos e mais); cor da pele autorrelatada (branca, preta, amarela, parda, indígena. $\mathrm{O}$ ambiente externo foi avaliado pela localização do domicílio (urbana, rural, distrito); se o idoso morava sozinho (sim, não); tempo de moradia no domicílio ( $\leq 10$ anos, $11-24$, 25-37, 38 anos e mais); condição de propriedade do domicilio (próprio, alugado, de parentes, outro); tipo de construção (alvenaria, outro); abastecimento de água (rede geral canalizada, poço ou nascente); presença de sanitário no domicílio (sim, não); rede de esgoto domiciliar (rede geral canalizada, fossa); coleta de lixo (serviço de limpeza público, outro); energia elétrica no domicilio (sim, não). A disponibilidade de recursos foi medida por meio do nível de escolaridade (analfabeto, 1o grau, 2o grau, graduado); renda mensal do idoso $(\leq \mathrm{R} \$ 622,00, \mathrm{R} \$ 622,00-820,00$, > R\$ 820,00); aposentado (sim, não); estado civil (casado, solteiro, viúvo, separado); participação em grupo para terceira idade (sim, não). O quarto nível reuniu as variáveis sobre os comportamentos de saúde: fuma ( $\operatorname{sim}$, não); ingere bebidas alcoólicas (sim, não); possui algum problema para dormir (sim, não); pratica atividade física (sim, não); teve perda de apetite nos últimos seis meses (sim, não); possui algum problema para mastigar ou deglutir os alimentos (sim, não); número de refeições diárias (uma, duas, três, quatro ou mais); ingestão diária de líquido (uma, duas, três, quatro ou mais); autoavaliação do peso (abaixo do peso, peso normal, acima do peso). As variáveis sobre o uso de serviços odontológicos estavam no penúltimo nível de análise: visita frequente ao dentista (sim, não); plano odontológico (sim, não); tipo de serviços de saúde bucal (público, particular, plano/convênio, outro); consulta ao dentista nos últimos seis meses (sim, 


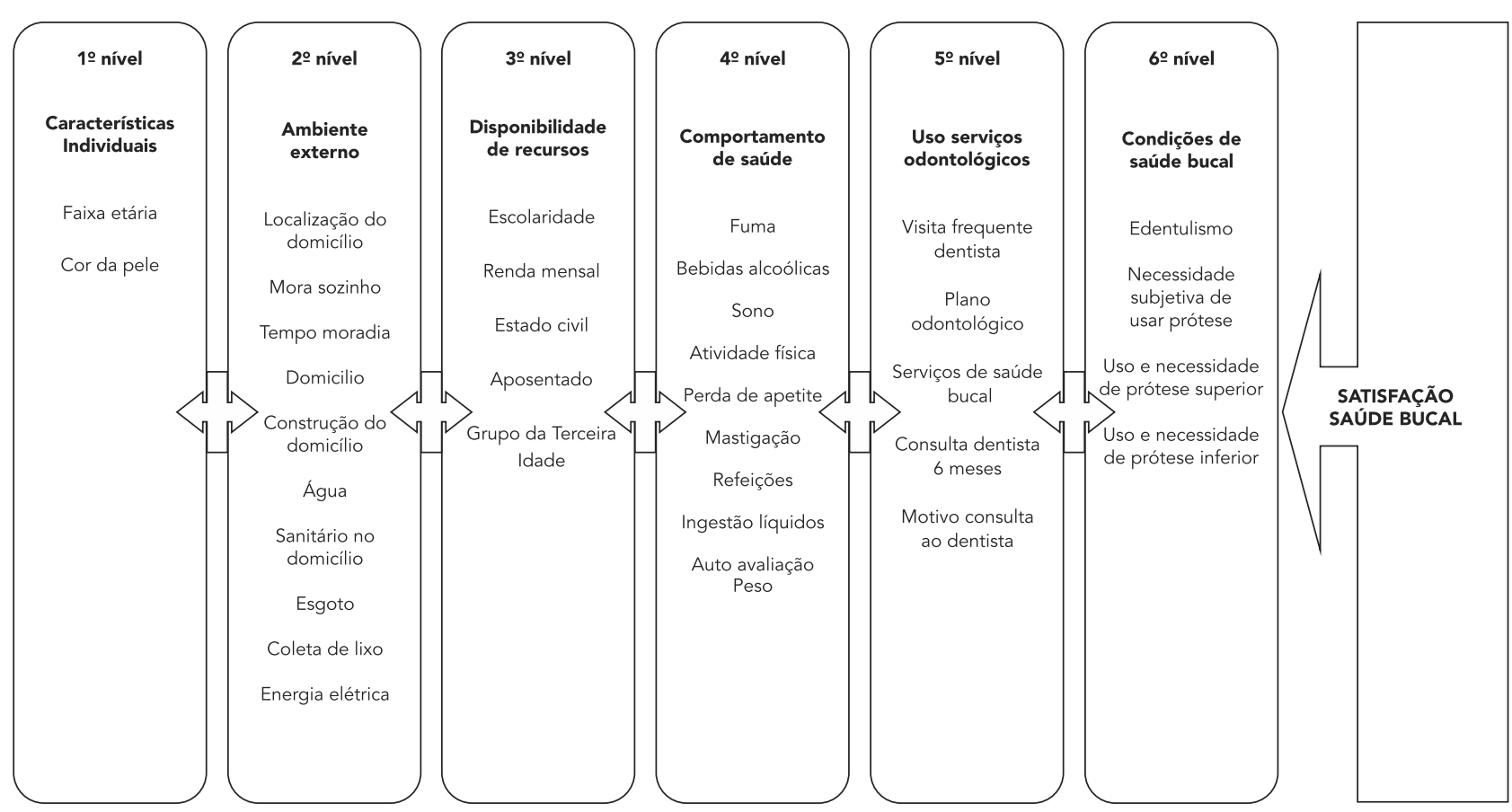

não); motivo de consulta ao dentista (dor, prevenção, tratamento, não consultou). Finalmente, no nível proximal ficaram as condições de saúde bucal: edentulismo (sim, não); autoavaliação da necessidade de usar/trocar prótese (sim, não); avaliação clínica do uso e necessidade de prótese superior (não usa e não necessita, não usa e necessita, usa e não necessita, usa e necessita); avaliação clínica do uso e necessidade de prótese inferior (não usa e não necessita, não usa e necessita, usa e não necessita, usa e necessita).

\section{Análise dos dados}

Inicialmente, realizou-se uma análise descritiva da amostra, estratificada por gênero. A análise de regressão de Poisson foi utilizada para estimar a prevalência de edentulismo por gênero e faixa etária. A satisfação bucal e a necessidade subjetiva de prótese para cada grupo (homens, mulheres e total) foram comparadas em relação ao edentulismo. Os resultados foram expressos como odds ratio (OR) e IC95\%.

Neste trabalho, optou-se por aplicar o modelo PLUM (polytomous universal model) que incorpora a natureza ordinal da variável dependente na análise. Sendo assim, foi realizada uma regressão logística com o modelo OR proporcional e função Logit. Recomenda-se executar uma análise univariada para seleção dos efeitos principais e incluir no modelo apenas as variáveis significativamente associadas ao desfecho com um nível de significância prefixado. Em seguida, as probabilidades entre as categorias da variável dependente são comparadas por meio do cálculo da OR bruta e ajustada 24. Para identificar os fatores associados à satisfação com saúde bucal, realizou-se uma análise univariada para cada variável independente e, posteriormente em cada bloco de associação. Dentro de cada nível hierárquico, as variáveis com $\mathrm{p}<0,20$ foram testadas em modelos múltiplos. Ao final, as variáveis com $\mathrm{p}<0,05$ permaneceram no modelo final de cada nível e foram consideradas fatores de ajuste para os blocos subsequentes. Para estimar as probabilidades do ponto de corte da variável dependente, o modelo construído apresenta limiares (thresholds) para as categorias inferiores (insatisfeito, nem insatisfeito nem satisfeito) em relação à de maior ordem 
(satisfeito). Para além dessa utilização, o seu interesse é reduzido 24

Considerando-se que a depressão e a ansiedade afetam a autoavaliação/percepção de saúde na população idosa 25 , foi realizado um ajuste no modelo final levando-se em conta a presença de depressão medida pela Escala Geriátrica de Depressão, versão curta com 15 itens (GDS-15) 26.

Todas as análises foram realizadas para homens e mulheres separadamente. Os testes de homogeneidade dos declives e de multicolinearidade foram realizados com ajuste de Pearson para analisar a validade do modelo construído. Para verificar a possível interferência do número reduzido de algumas observações, utilizou-se a análise dos resíduos para dados ordinais proposta por McCullagh 27. Todos esses testes evidenciaram que o modelo tinha todos os pressupostos satisfeitos. O efeito do desenho amostral complexo foi considerado em todas as análises realizadas, utilizando-se o software estatístico SPSS versão 18.0 (IBM SPSS, IBM Corp., Armonk, Estados Unidos).

\section{Resultados}

Dentre os domicílios sorteados para obter a amostra, 27 idosos haviam mudado de endereço, 38 não foram localizados e 35 faleceram no período da coleta de dados, totalizando uma perda de 4,8\%. Entre os idosos encontrados nos domicílios houve $5,8 \%$ de recusa e $1,2 \%$ de perda por outros motivos. Dos idosos entrevistados, 25 entrevistas foram realizadas com o cuidador devido a dificuldades físicas e/ou mentais, não tendo sido, por este motivo, incluídas nesta análise. Portanto, a amostra do presente estudo foi constituída por 2.052 indivíduos.

Em relação à satisfação com a saúde bucal, $25,9 \%$ dos idosos estavam insatisfeitos, $14,7 \%$ nem insatisfeitos nem satisfeitos e $59,4 \%$ satisfeitos. No grupo satisfeito com a saúde bucal (1.219 idosos), a maioria era do sexo feminino $(57,3 \%)$, tinha menos de 70 anos de idade $(65,7 \%)$, edêntula $(70,9 \%)$ e achava que não era necessário trocar/usar prótese $(72,8 \%)$ (Tabela 1 ).

$\mathrm{Na}$ análise por gênero, observou-se que a maioria dos idosos dos sexos masculino (63\%) e feminino (57\%) estava satisfeita com a saúde bucal. Em relação à faixa etária, os idosos mais velhos estavam mais satisfeitos para ambos os gêneros (Tabela 1).

A prevalência de edentulismo nos gêneros masculino e feminino foi de $63 \%$ e $68,8 \%$, respectivamente, sendo que houve diferença entre eles apenas na faixa etária de 65-69 anos $(\mathrm{RP}=1,13$; $\mathrm{p}=0,026)$. Evidenciou-se ainda um crescimento dessa prevalência devido ao aumento da idade, sendo $85,5 \%$ entre as mulheres e $66,1 \%$ para os homens com 80 anos e mais de idade. De acordo com o modelo de Poisson, não houve diferença de prevalência de edentulismo estatisticamente significante entre as faixas etárias para o gênero masculino. No gênero feminino, a prevalência de edentulismo nas faixas etárias 60-64 anos e 65-69 é 1,20 e 1,23 maior, respectivamente, quando em comparação com as idosas com 80 anos ou mais (Tabela 2).

Na análise univariada, a satisfação com a saúde bucal estabeleceu uma relação direta estatisticamente significante com a faixa etária dos idosos em ambos os gêneros, sendo que quanto mais velho o idoso mais satisfeito ele estava com a saúde bucal. Para o gênero masculino, a maioria das variáveis referentes ao ambiente externo, disponibilidade de recursos e uso serviços odontológicos não esteve associada à satisfação com a saúde bucal. Na análise por nível, verifica-se que para ambos os gêneros todas as variáveis referentes às condições de saúde bucal foram significantemente associadas ao desfecho (resultados não apresentados).

As Tabelas 3 e 4 revelaram que o modelo, para ambos os gêneros, foi formado por variáveis referentes a características individuais, ambiente externo, comportamentos de saúde e condições de saúde bucal. Para o gênero feminino, a variável sobre plano odontológico também permaneceu associada ao desfecho. A chance de o idoso edêntulo estar satisfeito com a saúde bucal é de $60 \%(\mathrm{p}=0,003)$ para homens e $55 \%$ ( $\mathrm{p}<0,001)$ para mulheres quando comparados com os idosos dentados. A autoavaliação de necessidade de prótese permaneceu inversamente associada ao desfecho para homens (OR $=5,08$; $\mathrm{p}=0,001)$ e mulheres (OR $=4,66 ; \mathrm{p}<0,001)$. Os idosos do gênero masculino que não usam e necessitam de algum tipo de prótese tiveram 2,54 mais chances de estar menos satisfeitos com a saúde bucal quando comparados àqueles que usam e necessitam trocar as próteses $(\mathrm{p}<0,001)$.

Os limiares que separam a categoria "insatisfeito" da "nem satisfeito nem insatisfeito" foram 2,4 e 2,2 vezes maiores em relação à categoria "satisfeito" para os gêneros masculino e feminino, respectivamente (Tabelas 3 e 4).

Após o ajuste pela variável depressão, a faixa etária e a presença de sanitário no domicílio foram excluídas no modelo final para o gênero masculino (Tabela 3). Em relação ao gênero feminino, cor da pele autorrelatada, autoavaliação do peso e número de refeições por dia não se mantiveram associadas ao desfecho no modelo ajustado (Tabela 4). 
Tabela 1

Descrição dos idosos por gênero em relação à satisfação com a saúde bucal, segundo características individuais, ambiente externo, disponibilidade de recursos, comportamentos de saúde, uso de serviços odontológicos e condições de saúde bucal. Sete Lagoas, Minas Gerais, Brasil, 2012.

\begin{tabular}{|c|c|c|c|c|c|c|c|c|c|c|c|c|}
\hline \multirow[t]{3}{*}{ Variáveis/Categorias } & \multicolumn{6}{|c|}{ Masculino $(\mathrm{N}=826)$} & \multicolumn{6}{|c|}{ Feminino $(N=1.226)$} \\
\hline & \multicolumn{2}{|c|}{$\begin{array}{l}\text { Insatisfeito } \\
(n=184)\end{array}$} & \multicolumn{2}{|c|}{$\begin{array}{c}\text { Nem insatisfeito } \\
\text { nem satisfeito } \\
(n=122)\end{array}$} & \multicolumn{2}{|c|}{$\begin{array}{l}\text { Satisfeito } \\
(n=520)\end{array}$} & \multicolumn{2}{|c|}{$\begin{array}{l}\text { Insatisfeito } \\
(n=347)\end{array}$} & \multicolumn{2}{|c|}{$\begin{array}{c}\text { Nem insatisfeito } \\
\text { nem satisfeito } \\
(n=180)\end{array}$} & \multicolumn{2}{|c|}{$\begin{array}{l}\text { Satisfeito } \\
(n=699)\end{array}$} \\
\hline & $\mathbf{n}$ & $\%$ & $\mathrm{n}$ & $\%$ & $\mathrm{n}$ & $\%$ & $\mathrm{n}$ & $\%$ & $\mathrm{n}$ & $\%$ & $\mathrm{n}$ & $\%$ \\
\hline \multicolumn{13}{|l|}{ Características individuais } \\
\hline \multicolumn{13}{|l|}{ Faixa etária (anos) } \\
\hline $60-64$ & 60 & 27,1 & 39 & 17,6 & 122 & 55,3 & 97 & 31,3 & 53 & 17,1 & 160 & 51,6 \\
\hline $65-69$ & 49 & 25,4 & 30 & 15,5 & 114 & 59,1 & 94 & 32,5 & 45 & 15,6 & 150 & 51,9 \\
\hline $70-74$ & 32 & 18,7 & 26 & 15,2 & 113 & 66,1 & 71 & 29,2 & 30 & 12,3 & 142 & 58,5 \\
\hline $75-79$ & 20 & 17,1 & 11 & 9,4 & 86 & 73,5 & 46 & 24,1 & 27 & 14,1 & 118 & 61,8 \\
\hline 80 e mais & 23 & 18,5 & 16 & 14,9 & 85 & 66,6 & 39 & 20,2 & 26 & 13,0 & 132 & 66,8 \\
\hline \multicolumn{13}{|l|}{ Cor da pele autorrelatada } \\
\hline Branca & 51 & 17,1 & 40 & 13,4 & 207 & 69,5 & 110 & 26,1 & 55 & 13,1 & 255 & 60,8 \\
\hline Preta & 36 & 29,0 & 14 & 11,3 & 74 & 59,7 & 55 & 32,5 & 28 & 16,6 & 86 & 50,9 \\
\hline Amarela & 87 & 23,5 & 64 & 17,3 & 220 & 59,2 & 160 & 28,5 & 91 & 15,6 & 325 & 55,9 \\
\hline Parda & 1 & 12,5 & 1 & 12,5 & 6 & 75,0 & 9 & 47,4 & 1 & 5,3 & 9 & 47,3 \\
\hline Indígena & 6 & 33,3 & 2 & 11,1 & 10 & 55,6 & 3 & 13,0 & 4 & 17,4 & 16 & 69,6 \\
\hline \multicolumn{13}{|l|}{ Ambiente externo } \\
\hline \multicolumn{13}{|l|}{ Localização do domicílio } \\
\hline Urbana & 174 & 24,0 & 95 & 13,1 & 455 & 62,9 & 316 & 29,0 & 154 & 14,1 & 619 & 56,9 \\
\hline Rural & 7 & 8,9 & 26 & 32,9 & 46 & 58,2 & 18 & 16,8 & 22 & 20,6 & 67 & 62,6 \\
\hline Distrito & 3 & 13,0 & 1 & 4,3 & 19 & 82,7 & 13 & 43,3 & 4 & 13,3 & 13 & 43,3 \\
\hline \multicolumn{13}{|l|}{ Mora sozinho } \\
\hline $\operatorname{Sim}$ & 24 & 30,4 & 10 & 12,7 & 45 & 56,9 & 58 & 29,3 & 27 & 13,6 & 113 & 57,1 \\
\hline Não & 158 & 21,2 & 112 & 15,0 & 475 & 63,8 & 287 & 28,0 & 152 & 14,8 & 586 & 57,2 \\
\hline \multicolumn{13}{|l|}{ Tempo moradia (anos) } \\
\hline$\leq 10$ & 62 & 28,7 & 33 & 15,3 & 123 & 56,0 & 85 & 27,9 & 49 & 16,1 & 171 & 56,0 \\
\hline $11-24$ & 50 & 23,1 & 30 & 13,9 & 136 & 63,0 & 93 & 30,9 & 48 & 15,9 & 160 & 53,2 \\
\hline $25-37$ & 43 & 21,9 & 27 & 13,8 & 126 & 64,3 & 99 & 31,8 & 50 & 15,9 & 164 & 52,2 \\
\hline 38 e mais & 29 & 14,6 & 32 & 16,2 & 137 & 69,2 & 70 & 22,4 & 35 & 11,2 & 207 & 66,3 \\
\hline \multicolumn{13}{|l|}{ Domicilio } \\
\hline Próprio & 156 & 21,1 & 112 & 15,2 & 471 & 63,7 & 308 & 28,2 & 155 & 14,2 & 631 & 57,6 \\
\hline Alugado & 13 & 39,4 & 3 & 9,1 & 17 & 51,5 & 25 & 37,3 & 10 & 14,9 & 32 & 47,8 \\
\hline De parentes & 8 & 22,2 & 3 & 8,3 & 25 & 69,5 & 12 & 20,1 & 14 & 24,6 & 31 & 54,5 \\
\hline Outro & 7 & 38,9 & 4 & 22,2 & 7 & 38,9 & 2 & 25,0 & 1 & 12,5 & 5 & 62,5 \\
\hline \multicolumn{13}{|l|}{ Construção do domicílio } \\
\hline Alvenaria (tijolo) & 184 & 22,3 & 122 & 14,8 & 520 & 62,9 & 347 & 28,4 & 179 & 14,6 & 696 & 57,0 \\
\hline Outro & 0 & 0,0 & 0 & 0,0 & 0 & 0,0 & 0 & 0,0 & 1 & 25,0 & 3 & 75,0 \\
\hline \multicolumn{13}{|l|}{ Abastecimento de água } \\
\hline Rede geral canalizada & 180 & 22,3 & 116 & 14,4 & 510 & 63,3 & 344 & 28,4 & 177 & 14,7 & 683 & 56,9 \\
\hline Poço ou nascente & 4 & 20,0 & 6 & 30,0 & 10 & 50,0 & 3 & 13,6 & 3 & 13,6 & 16 & 72,8 \\
\hline \multicolumn{13}{|l|}{ Sanitário no domicílio } \\
\hline $\operatorname{Sim}$ & 183 & 22,4 & 122 & 14,9 & 513 & 62,7 & 347 & 28,4 & 180 & 14,7 & 695 & 56,9 \\
\hline Não & 1 & 12,5 & 0 & 0,0 & 07 & 87,5 & 0 & 0,0 & 0 & 0,0 & 4 & 100,0 \\
\hline Esgoto & & & & & & & & & & & & \\
\hline Rede geral canalizada & 175 & 23,0 & 100 & 13,1 & 480 & 63,9 & 328 & 28,3 & 160 & 13,8 & 670 & 57,7 \\
\hline Fossa & 9 & 13,8 & 22 & 33,8 & 34 & 52,4 & 19 & 27,9 & 20 & 29,4 & 29 & 42,6 \\
\hline
\end{tabular}

(continua) 
Tabela 1 (continuação)

\begin{tabular}{|c|c|c|c|c|c|c|c|c|c|c|c|c|}
\hline \multirow[t]{3}{*}{ Variáveis/Categorias } & \multicolumn{6}{|c|}{ Masculino ( $N=826)$} & \multicolumn{6}{|c|}{ Feminino $(\mathrm{N}=1.226)$} \\
\hline & \multicolumn{2}{|c|}{$\begin{array}{l}\text { Insatisfeito } \\
(n=184)\end{array}$} & \multicolumn{2}{|c|}{$\begin{array}{c}\text { Nem insatisfeito } \\
\text { nem satisfeito } \\
(n=122)\end{array}$} & \multicolumn{2}{|c|}{$\begin{array}{l}\text { Satisfeito } \\
(n=520)\end{array}$} & \multicolumn{2}{|c|}{$\begin{array}{l}\text { Insatisfeito } \\
(n=347)\end{array}$} & \multicolumn{2}{|c|}{$\begin{array}{c}\text { Nem insatisfeito } \\
\text { nem satisfeito } \\
(n=180)\end{array}$} & \multicolumn{2}{|c|}{$\begin{array}{l}\text { Satisfeito } \\
(n=699)\end{array}$} \\
\hline & $\mathbf{n}$ & $\%$ & $\mathbf{n}$ & $\%$ & $\mathbf{n}$ & $\%$ & $\mathbf{n}$ & $\%$ & $\mathrm{n}$ & $\%$ & $\mathbf{n}$ & $\%$ \\
\hline \multicolumn{13}{|l|}{ Ambiente externo } \\
\hline \multicolumn{13}{|l|}{ Coleta de lixo } \\
\hline Serviço de limpeza & 179 & 22,2 & 119 & 14,9 & 502 & 62,9 & 343 & 28,6 & 175 & 14,6 & 682 & 56,8 \\
\hline Outro & 5 & 19,2 & 3 & 11,5 & 18 & 69,3 & 4 & 15,4 & 5 & 19,2 & 17 & 65,4 \\
\hline \multicolumn{13}{|l|}{ Energia elétrica } \\
\hline Sim & 166 & 22,6 & 114 & 15,5 & 454 & 61,9 & 319 & 29,2 & 170 & 15,5 & 605 & 55,3 \\
\hline Não & 18 & 19,1 & 9 & 9,6 & 67 & 71,3 & 28 & 21,2 & 10 & 7,6 & 94 & 71,2 \\
\hline \multicolumn{13}{|c|}{ Disponibilidade de recursos } \\
\hline \multicolumn{13}{|l|}{ Escolaridade } \\
\hline Analfabeto & 53 & 22,1 & 32 & 13,3 & 155 & 64,6 & 92 & 27,1 & 51 & 15,0 & 196 & 57,9 \\
\hline 1ㅇ grau & 113 & 22,6 & 79 & 15,8 & 308 & 61,6 & 231 & 29,5 & 109 & 13,9 & 442 & 56,6 \\
\hline 2o grau & 13 & 20,6 & 10 & 15,9 & 40 & 63,5 & 18 & 26,9 & 13 & 19,4 & 36 & 53,7 \\
\hline Graduado & 5 & 21,7 & 1 & 4,3 & 17 & 74,0 & 6 & 15,8 & 7 & 18,4 & 25 & 65,8 \\
\hline \multicolumn{13}{|l|}{ Renda mensal (R\$) } \\
\hline$\leq 622,00$ & 115 & 23,5 & 75 & 15,3 & 300 & 61,2 & 243 & 27,7 & 137 & 15,6 & 497 & 56,4 \\
\hline $622,00-820,00$ & 21 & 28,0 & 14 & 18,7 & 40 & 53,3 & 31 & 30,1 & 14 & 13,6 & 58 & 56,3 \\
\hline$>820,00$ & 49 & 18,1 & 37 & 13,7 & 185 & 68,2 & 73 & 29,7 & 29 & 11,8 & 144 & 58,9 \\
\hline \multicolumn{13}{|l|}{ Estado civil } \\
\hline Casado & 119 & 19,4 & 95 & 15,6 & 401 & 65,0 & 130 & 27,7 & 66 & 14,1 & 273 & 58,2 \\
\hline Solteiro & 14 & 22,2 & 12 & 19,0 & 34 & 58,8 & 42 & 30,0 & 31 & 22,1 & 67 & 47,9 \\
\hline Viúvo & 34 & 36,6 & 12 & 12,9 & 47 & 50,5 & 148 & 28,5 & 67 & 12,9 & 304 & 58,6 \\
\hline Separado & 17 & 28,8 & 4 & 6,8 & 38 & 64,4 & 25 & 26,3 & 16 & 16,8 & 54 & 57,0 \\
\hline \multicolumn{13}{|l|}{ Aposentado } \\
\hline Sim & 148 & 21,2 & 104 & 14,6 & 456 & 64,2 & 222 & 27,1 & 126 & 15,4 & 471 & 57,5 \\
\hline Não & 36 & 28,3 & 22 & 17,3 & 69 & 54,4 & 125 & 30,7 & 54 & 13,3 & 228 & 56,0 \\
\hline \multicolumn{13}{|c|}{ Grupo da terceira idade } \\
\hline Sim & 6 & 14,6 & 6 & 14,6 & 29 & 70,8 & 38 & 32,8 & 20 & 17,2 & 58 & 50,0 \\
\hline Não & 179 & 22,7 & 116 & 14,8 & 496 & 62,5 & 309 & 27,6 & 160 & 14,4 & 641 & 57,7 \\
\hline \multicolumn{13}{|c|}{ Comportamentos de saúde } \\
\hline Fuma & & & & & & & & & & & & \\
\hline Sim & 40 & 28,0 & 25 & 17,5 & 78 & 54,5 & 30 & 36,6 & 8 & 9,8 & 44 & 53,6 \\
\hline Não & 144 & 21,1 & 97 & 14,8 & 442 & 64,1 & 317 & 27,7 & 172 & 15,0 & 655 & 57,3 \\
\hline Ingere bebidas alcoóli & & & & & & & & & & & & \\
\hline Sim & 61 & 23,7 & 36 & 14,0 & 160 & 62,3 & 37 & 29,1 & 21 & 16,5 & 68 & 54,4 \\
\hline Não & 123 & 21,6 & 86 & 15,1 & 360 & 63,3 & 310 & 28,2 & 159 & 14,5 & 631 & 57,3 \\
\hline Problema para dormir & & & & & & & & & & & & \\
\hline $\operatorname{Sim}$ & 67 & 26,0 & 42 & 16,3 & 149 & 57,7 & 179 & 34,3 & 82 & 15,7 & 261 & 50,0 \\
\hline Não & 117 & 20,6 & 80 & 14,1 & 371 & 65,3 & 168 & 23,9 & 98 & 13,9 & 438 & 62,2 \\
\hline Prática de atividade fís & & & & & & & & & & & & \\
\hline Sim & 42 & 19,4 & 33 & 15,3 & 141 & 65,3 & 97 & 29,5 & 45 & 13,7 & 187 & 56,8 \\
\hline Não & 142 & 23,3 & 89 & 14,6 & 379 & 62,1 & 250 & 27,9 & 135 & 15,1 & 512 & 57,0 \\
\hline Perda de apetite & & & & & & & & & & & & \\
\hline Sim & 56 & 31,3 & 28 & 15,6 & 95 & 53,1 & 142 & 36,4 & 61 & 15,6 & 187 & 48,0 \\
\hline Não & 128 & 19,8 & 94 & 14,5 & 425 & 65,7 & 205 & 24,5 & 119 & 14,2 & 512 & 61,3 \\
\hline
\end{tabular}

(continua) 
Tabela 1 (continuação)

\begin{tabular}{|c|c|c|c|c|c|c|c|c|c|c|c|c|}
\hline \multirow[t]{3}{*}{ Variáveis/Categorias } & \multicolumn{6}{|c|}{ Masculino $(\mathrm{N}=826)$} & \multicolumn{6}{|c|}{ Feminino $(\mathrm{N}=1.226)$} \\
\hline & \multicolumn{2}{|c|}{$\begin{array}{l}\text { Insatisfeito } \\
(n=184)\end{array}$} & \multicolumn{2}{|c|}{$\begin{array}{c}\text { Nem insatisfeito } \\
\text { nem satisfeito } \\
(n=122)\end{array}$} & \multicolumn{2}{|c|}{$\begin{array}{l}\text { Satisfeito } \\
(n=520)\end{array}$} & \multicolumn{2}{|c|}{$\begin{array}{l}\text { Insatisfeito } \\
(n=347)\end{array}$} & \multicolumn{2}{|c|}{$\begin{array}{c}\text { Nem insatisfeito } \\
\text { nem satisfeito } \\
(n=180)\end{array}$} & \multicolumn{2}{|c|}{$\begin{array}{l}\text { Satisfeito } \\
(n=699)\end{array}$} \\
\hline & $\mathbf{n}$ & $\%$ & $\mathbf{n}$ & $\%$ & $\mathbf{n}$ & $\%$ & $\mathbf{n}$ & $\%$ & $n$ & $\%$ & $\mathbf{n}$ & $\%$ \\
\hline \multicolumn{13}{|c|}{ Comportamentos de saúde } \\
\hline \multicolumn{13}{|c|}{ Problema para mastigar ou } \\
\hline \multicolumn{13}{|c|}{ deglutir } \\
\hline Sim & 28 & 37,8 & 18 & 24,3 & 28 & 37,9 & 94 & 50,8 & 21 & 11,4 & 70 & 37,8 \\
\hline Não & 156 & 20,7 & 104 & 13,8 & 492 & 65,5 & 253 & 24,3 & 159 & 15,3 & 629 & 60,4 \\
\hline \multicolumn{13}{|l|}{ Refeições por dia } \\
\hline 1 & 7 & 24,1 & 3 & 10,3 & 19 & 65,6 & 26 & 48,1 & 6 & 11,1 & 22 & 40,8 \\
\hline 2 & 56 & 28,0 & 22 & 11,0 & 122 & 61,0 & 61 & 29,3 & 25 & 12,0 & 122 & 58,7 \\
\hline 3 & 57 & 19,9 & 43 & 15,0 & 187 & 65,1 & 102 & 24,0 & 60 & 14,1 & 263 & 61,9 \\
\hline 4 ou mais & 64 & 20,8 & 53 & 17,2 & 191 & 62,0 & 156 & 29,2 & 89 & 16,7 & 289 & 54,1 \\
\hline \multicolumn{13}{|c|}{ Copos de líquido por dia } \\
\hline 1 & 9 & 26,5 & 4 & 11,8 & 21 & 61,7 & 18 & 34,6 & 3 & 5,8 & 31 & 59,6 \\
\hline 2 & 18 & 23,7 & 13 & 17,1 & 45 & 59,2 & 33 & 27,5 & 18 & 15,0 & 69 & 57,5 \\
\hline 3 & 26 & 19,0 & 19 & 13,9 & 92 & 67,1 & 53 & 23,8 & 36 & 16,1 & 135 & 60,1 \\
\hline 4 ou mais & 131 & 22,6 & 86 & 14,9 & 362 & 62,5 & 242 & 29,3 & 123 & 14,9 & 462 & 55,8 \\
\hline \multicolumn{13}{|c|}{ Autoavaliação do peso } \\
\hline Abaixo do peso & 20 & 24,1 & 16 & 19,3 & 47 & 56,6 & 40 & 32,2 & 24 & 19,4 & 60 & 48,4 \\
\hline No peso normal & 117 & 20,2 & 81 & 14,0 & 381 & 65,8 & 175 & 24,2 & 101 & 14,0 & 447 & 61,8 \\
\hline Acima do peso & 47 & 28,8 & 25 & 15,3 & 91 & 55,9 & 132 & 34,9 & 55 & 14,6 & 191 & 50,5 \\
\hline \multicolumn{13}{|c|}{ Uso de serviços odontológicos } \\
\hline \multicolumn{13}{|c|}{ Visita frequente ao dentista } \\
\hline $\operatorname{Sim}$ & 24 & 16,8 & 22 & 15,4 & 97 & 67,8 & 75 & 30,2 & 33 & 13,3 & 140 & 56,5 \\
\hline Não & 160 & 23,4 & 100 & 14,6 & 423 & 62,0 & 272 & 27,8 & 147 & 15,0 & 559 & 57,2 \\
\hline \multicolumn{13}{|l|}{ Plano odontológico } \\
\hline $\operatorname{Sim}$ & 13 & 16,9 & 17 & 21,1 & 47 & 62,0 & 33 & 22,8 & 19 & 13,3 & 93 & 63,9 \\
\hline Não & 171 & 22,8 & 105 & 14,4 & 473 & 62,0 & 316 & 29,0 & 161 & 14,9 & 606 & 56,1 \\
\hline \multicolumn{13}{|c|}{ Serviços de saúde bucal } \\
\hline Público & 84 & 29,3 & 54 & 18,8 & 149 & 51,9 & 139 & 34,1 & 71 & 17,4 & 198 & 48,5 \\
\hline Particular & 79 & 21,0 & 45 & 12,1 & 248 & 66,9 & 151 & 26,9 & 76 & 13,5 & 335 & 59,6 \\
\hline Plano/Convênio & 5 & 10,6 & 12 & 25,5 & 30 & 63,9 & 21 & 23,9 & 15 & 17,0 & 52 & 59,1 \\
\hline Outro & 17 & 14,0 & 11 & 9,3 & 93 & 76,7 & 36 & 21,4 & 18 & 10,7 & 114 & 67,9 \\
\hline \multicolumn{13}{|c|}{ Consulta dentista 6 meses } \\
\hline $\operatorname{Sim}$ & 42 & 22,8 & 27 & 14,7 & 115 & 62,5 & 99 & 30,9 & 44 & 13,8 & 177 & 55,3 \\
\hline Não & 142 & 22,1 & 95 & 14,8 & 408 & 63,1 & 248 & 27,4 & 136 & 15,0 & 522 & 57,6 \\
\hline Motivo da consulta & & & & & & & & & & & & \\
\hline Dor & 35 & 42,7 & 14 & 17,1 & 33 & 40,2 & 44 & 38,3 & 16 & 13,5 & 55 & 48,2 \\
\hline Prevenção & 15 & 20,3 & 6 & 8,1 & 53 & 71,6 & 23 & 22,1 & 17 & 16,3 & 64 & 61,6 \\
\hline Tratamento & 84 & 18,7 & 58 & 12,9 & 307 & 68,4 & 202 & 27,7 & 96 & 13,2 & 430 & 59,1 \\
\hline Não consultou & 50 & 22,6 & 44 & 19,5 & 127 & 57,9 & 78 & 28,0 & 51 & 18,3 & 150 & 53,7 \\
\hline Condições de saúde b & & & & & & & & & & & & \\
\hline Edentulismo & & & & & & & & & & & & \\
\hline $\operatorname{Sim}$ & 100 & 19,2 & 70 & 13,5 & 350 & 67,3 & 211 & 25,0 & 119 & 14,1 & 513 & 60,9 \\
\hline Não & 83 & 27,2 & 52 & 17,0 & 170 & 55,9 & 136 & 35,6 & 61 & 16,0 & 185 & 48,4 \\
\hline
\end{tabular}

(continua) 
Tabela 1 (continuação)

\begin{tabular}{|c|c|c|c|c|c|c|c|c|c|c|c|c|}
\hline \multirow[t]{3}{*}{ Variáveis/Categorias } & \multicolumn{6}{|c|}{ Masculino $(\mathrm{N}=826)$} & \multicolumn{6}{|c|}{ Feminino $(\mathrm{N}=1.226)$} \\
\hline & \multicolumn{2}{|c|}{$\begin{array}{l}\text { Insatisfeito } \\
(n=184)\end{array}$} & \multicolumn{2}{|c|}{$\begin{array}{l}\text { Nem insatisfeito } \\
\text { nem satisfeito } \\
(n=122)\end{array}$} & \multicolumn{2}{|c|}{$\begin{array}{l}\text { Satisfeito } \\
(n=520)\end{array}$} & \multicolumn{2}{|c|}{$\begin{array}{l}\text { Insatisfeito } \\
(n=347)\end{array}$} & \multicolumn{2}{|c|}{$\begin{array}{c}\text { Nem insatisfeito } \\
\text { nem satisfeito } \\
(n=180)\end{array}$} & \multicolumn{2}{|c|}{$\begin{array}{l}\text { Satisfeito } \\
(\mathrm{n}=699)\end{array}$} \\
\hline & $\mathrm{n}$ & $\%$ & $\mathrm{n}$ & $\%$ & $\mathrm{n}$ & $\%$ & $\mathrm{n}$ & $\%$ & $n$ & $\%$ & $\mathrm{n}$ & $\%$ \\
\hline \multicolumn{13}{|l|}{ Condições de saúde bucal } \\
\hline \multicolumn{13}{|l|}{$\begin{array}{l}\text { Necessidade subjetiva de usar } \\
\text { prótese }\end{array}$} \\
\hline Sim & 124 & 44,4 & 43 & 15,4 & 112 & 40,2 & 246 & 48,1 & 77 & 15,1 & 188 & 36,8 \\
\hline Não & 60 & 11,0 & 79 & 14,4 & 408 & 74,6 & 101 & 14,1 & 103 & 14,4 & 511 & 71,5 \\
\hline \multicolumn{13}{|l|}{$\begin{array}{l}\text { Uso e necessidade de prótese } \\
\text { superior }\end{array}$} \\
\hline Não usa e não necessita & 10 & 13,0 & 12 & 15,6 & 55 & 71,4 & 23 & 25,8 & 17 & 19,1 & 49 & 55,1 \\
\hline Não usa e necessita & 87 & 38,2 & 33 & 14,5 & 108 & 47,3 & 92 & 38,3 & 37 & 15,4 & 111 & 46,3 \\
\hline Usa e não necessita & 35 & 10,9 & 45 & 14,1 & 240 & 75,0 & 79 & 15,7 & 62 & 12,4 & 361 & 71,9 \\
\hline Usa e necessita & 50 & 25,1 & 32 & 16,1 & 117 & 58,8 & 153 & 39,0 & 64 & 16,3 & 175 & 44,7 \\
\hline \multicolumn{13}{|l|}{ Uso e necessidade de prótese } \\
\hline \multicolumn{13}{|l|}{ inferior } \\
\hline Não usa e não necessita & 14 & 12,6 & 20 & 18,0 & 77 & 69,4 & 38 & 25,9 & 26 & 17,7 & 83 & 56,4 \\
\hline Não usa e necessita & 104 & 34,0 & 47 & 15,4 & 155 & 50,6 & 147 & 38,1 & 63 & 16,3 & 176 & 45,6 \\
\hline Usa e não necessita & 21 & 8,5 & 33 & 13,4 & 192 & 78,1 & 50 & 13,0 & 45 & 11,7 & 291 & 75,3 \\
\hline Usa e necessita & 44 & 27,2 & 22 & 13,6 & 96 & 59,2 & 112 & 36,6 & 46 & 15,0 & 148 & 48,4 \\
\hline
\end{tabular}

\section{Discussão}

Devido à sua importância para avaliar os aspectos subjetivos de saúde bucal, a autoavaliação deste quesito é uma medida importante em estudos de base populacional 28. No Brasil, a satisfação ganhou destaque no projeto SBBrasil 2010, sendo este o principal motivo para usarmos esta variável no presente estudo 1 .

Os resultados mais importantes deste trabalho referem à análise da associação estatisticamente significante entre gênero e satisfação com a saúde bucal ( $p=0,007$ ), sendo que as mulheres estavam menos satisfeitas quando comparadas aos homens. Esses resultados corroboram os dados da Pesquisa Mundial de Saúde 16. Bulgarelli \& Manço 29 relataram maior satisfação com a saúde bucal entre os idosos mais velhos, mas sem associação significante com o gênero.

Por outro lado, a maioria dos idosos de ambos os gêneros estava satisfeita com a própria saúde bucal, ainda que as condições clínicas observadas tenham mostrado precariedade, com alta prevalência de perda dentária e necessidade de tratamento odontológico restaurador e/ou reabilitador. Identificou-se um estudo que avaliou a satisfação com saúde bucal na população idosa brasileira, mostrando que $61,5 \%$ dos idosos esta- vam satisfeitos e/ou muito satisfeitos em relação à própria saúde bucal 29.

Outros estudos têm mostrado boa autopercepção 3,5,6 e autoavaliação positiva da saúde bucal em idosos 9,10 associadas a condições de saúde bucal precárias. Esses resultados indicam que o idoso percebe sua saúde bucal de maneira peculiar, refletindo atitude de resignação culturalmente difundida ${ }^{12}$, especialmente quando comparados aos adultos 11 .

$\mathrm{Na}$ análise univariada, os idosos com menos de 70 anos estavam menos satisfeitos com a saúde bucal quando comparados aos octogenários. Entretanto, essa associação apenas foi mantida para os idosos com 60-64 anos do gênero masculino no modelo não ajustado. Os resultados em relação à idade são controversos. Alguns trabalhos relataram que os idosos têm uma avaliação melhor do que os mais jovens 11, outros observaram uma relação inversa 25 e alguns não encontraram nenhuma associação significativa 10,12. Para Bulgarelli \& Manço 29, os mais idosos aceitam mais facilmente os problemas da cavidade bucal como inevitáveis e, neste contexto antagônico, relatam maior satisfação com a saúde bucal.

Neste estudo as mulheres pardas tiveram uma menor satisfação com a saúde bucal na análise por nível, mas não no modelo final ajustado. 
Análise do modelo de regressão de Poisson para a prevalência de edentulismo em idosos segundo gênero e faixa etária. Sete Lagoas, Minas Gerais, Brasil, 2012.

\begin{tabular}{|c|c|c|c|c|c|c|c|c|c|c|}
\hline \multirow[t]{3}{*}{ Variáveis } & \multicolumn{6}{|c|}{ Edentulismo } & \multirow[t]{3}{*}{ RP } & \multicolumn{2}{|c|}{ IC95\% } & \multirow{3}{*}{$\begin{array}{l}\text { Valor } \\
\text { de } p\end{array}$} \\
\hline & \multicolumn{2}{|c|}{ Sim } & \multicolumn{2}{|c|}{ Não } & \multicolumn{2}{|c|}{ Total } & & \multirow[t]{2}{*}{ Início } & \multirow[t]{2}{*}{ Final } & \\
\hline & $\mathbf{n}$ & $\%$ & $\mathbf{n}$ & $\%$ & $\mathbf{n}$ & $\%$ & & & & \\
\hline \multicolumn{11}{|l|}{ Masculino } \\
\hline \multicolumn{11}{|c|}{ Faixa etária (anos) } \\
\hline $60-64$ & 109 & 49,3 & 112 & 50,7 & 221 & 100,0 & 0,98 & 0,80 & 1,20 & 0,839 \\
\hline $65-69$ & 116 & 60,1 & 77 & 39,9 & 193 & 100,0 & 0,95 & 0,82 & 1,11 & 0,522 \\
\hline $70-74$ & 120 & 70,6 & 50 & 29,4 & 170 & 100,0 & 0,99 & 0,85 & 1,17 & 0,929 \\
\hline $75-79$ & 93 & 79,5 & 24 & 20,5 & 117 & 100,0 & 0,87 & 0,74 & 1,02 & 0,090 \\
\hline 80 e mais & 82 & 66,1 & 42 & 33,9 & 124 & 100,0 & 1,00 & & & \\
\hline \multicolumn{11}{|l|}{ Feminino } \\
\hline \multicolumn{11}{|c|}{ Faixa etária (anos) } \\
\hline $60-64$ & 173 & 56,0 & 136 & 44,0 & 309 & 100,0 & 1,19 & 1,07 & 1,33 & 0,009 \\
\hline $65-69$ & 185 & 64,0 & 104 & 36,0 & 289 & 100,0 & 1,19 & 1,04 & 1,35 & 0,003 \\
\hline $70-74$ & 175 & 72,0 & 68 & 28,0 & 243 & 100,0 & 1,23 & 1,07 & 1,41 & 0,917 \\
\hline $75-79$ & 145 & 75,9 & 46 & 24,1 & 191 & 100,0 & 1,01 & 0,88 & 1,15 & 0,138 \\
\hline 80 e mais & 165 & 85,5 & 28 & 14,5 & 193 & 100,0 & 1,00 & & & \\
\hline \multicolumn{11}{|l|}{ Total } \\
\hline \multicolumn{11}{|c|}{ Faixa etária (anos) } \\
\hline $60-64$ & 282 & 53,2 & 248 & 46,8 & 530 & 100,0 & 1,10 * & 0,97 & 1,26 & 0,133 \\
\hline $65-69$ & 301 & 62,4 & 181 & 37,6 & 482 & 100,0 & 1,13 * & 1,01 & 1,26 & 0,026 \\
\hline $70-74$ & 295 & 71,4 & 118 & 28,6 & 413 & 100,0 & 1,00 * & 0,90 & 1,11 & 0,976 \\
\hline $75-79$ & 238 & 77,3 & 70 & 22,7 & 308 & 100,0 & 1,04 * & 0,93 & 1,17 & 0,517 \\
\hline 80 e mais & 247 & 77,9 & 70 & 22,1 & 317 & 100,0 & 1,00 & & & \\
\hline
\end{tabular}

IC95\%: intervalo de 95\% de confiança; RP: razão de prevalência.

* Razão de prevalência entre os gêneros por faixa etária, considerando o gênero masculino como referência.

De acordo com Martins et al. 10, a autoavaliação da saúde bucal é mais positiva entre os indivíduos brancos, resultados que devem ser levados em consideração no planejamento das ações em saúde.

Em relação às condições de moradia, os idosos do gênero masculino residentes na área urbana estavam menos satisfeitos quando comparados àqueles que vivem no distrito. Uma possível explicação para esses achados é que o meio social interfere de forma diferenciada na maneira pela qual os indivíduos pensam, sentem e agem a respeito de sua saúde 12. Martins et al. 23 encontraram associação negativa entre residir no meio rural e autopercepção boa de saúde bucal de idosos.

A falta de saneamento básico estava ligada a piores condições de saúde geral, porém este é um dos poucos estudos que investigou a relação entre condições e moradia e satisfação com saúde bucal.
$\mathrm{O}$ acesso à rede de esgoto se manteve associada à satisfação com saúde bucal de maneira inversa para ambos os gêneros. Os idosos que não tinham rede canalizada de esgoto têm $72 \%$ e $42 \%$ mais chances de relatarem menor satisfação com a saúde bucal para os gêneros masculino e feminino, respectivamente. Uma possível explicação para esses achados pode estar relacionada às diferenças estatisticamente significantes entre localização do domicílio (urbana, rural, distrito) e as condições de moradia (resultados não apresentados). O sanitário da maioria dos domicílios na área rural do município estava ligado à fossa (60,9\%), em 50\% estava localizado fora do domicílio e a fonte de água vem de poço ou nascente. Levando-se em conta que outras variáveis sobre saneamento coletadas no presente estudo (abastecimento de água, presença de sanitário e energia elétrica no domicílio, coleta de lixo) não foram associadas ao desfecho, não é possível garantir uma relação 
Tabela 3

Modelo de regressão ordinal final para a satisfação com a saúde bucal para o gênero masculino segundo características individuais, ambiente externo, disponibilidade de recursos, comportamentos de saúde, uso de serviços odontológicos e condições de saúde bucal. Sete Lagoas, Minas Gerais, Brasil, 2012.

\begin{tabular}{|c|c|c|c|c|c|c|}
\hline \multirow[t]{2}{*}{ Variáveis } & \multicolumn{3}{|c|}{ Modelo bruto } & \multicolumn{3}{|c|}{ Modelo ajustado * } \\
\hline & OR & IC95\% & $\begin{array}{l}\text { Valor } \\
\text { de } p\end{array}$ & OR & IC95\% & $\begin{array}{l}\text { Valor } \\
\text { de } p\end{array}$ \\
\hline \multicolumn{7}{|l|}{ Limiares do modelo ordinal } \\
\hline Insatisfeito & 9,80 & $1,09-10,76$ & $<0,001$ & 7,31 & $1,89-28,23$ & 0,004 \\
\hline Nem insatisfeito nem satisfeito & 3,89 & $1,44-4,51$ & 0,003 & 3,05 & $0,79-11,72$ & 0,010 \\
\hline \multicolumn{7}{|l|}{ Características individuais } \\
\hline \multicolumn{7}{|l|}{ Faixa etária (anos) } \\
\hline $60-64$ & 1,87 & $1,13-3,12$ & 0,016 & 1,43 & $0,88-2,33$ & 0,150 \\
\hline $65-69$ & 1,64 & $0,98-2,76$ & 0,061 & 1,30 & $0,79-2,14$ & 0,302 \\
\hline $70-74$ & 1,33 & $0,78-2,28$ & 0,301 & 1,03 & $0,61-1,73$ & 0,912 \\
\hline $75-79$ & 1,08 & $0,59-1,97$ & 0,808 & 1,24 & $0,69-2,22$ & 0,471 \\
\hline 80 e mais & 1,00 & & . & 1,00 & & \\
\hline \multicolumn{7}{|l|}{ Ambiente externo } \\
\hline \multicolumn{7}{|l|}{ Localização do domicílio } \\
\hline Urbana & 4,99 & $1,22-20,41$ & 0,025 & 4,24 & $1,10-16,31$ & 0,036 \\
\hline Rural & 2,76 & $0,76-9,99$ & 0,122 & 2,48 & $0,72-8,51$ & 0,150 \\
\hline Distrito & 1,00 & & & 1,00 & & \\
\hline \multicolumn{7}{|l|}{ Sanitário no domicílio } \\
\hline Sim & 7,68 & $0,86-68,13$ & 0,067 & & & \\
\hline Não & 1,00 & & & & & \\
\hline \multicolumn{7}{|l|}{ Esgoto } \\
\hline Rede geral canalizada & 0,19 & $0,08-0,51$ & 0,001 & 0,28 & $0,12-0,68$ & 0,005 \\
\hline Fossa & 1,00 & & . & 1,00 & & \\
\hline \multicolumn{7}{|l|}{ Comportamentos de saúde } \\
\hline \multicolumn{7}{|l|}{ Perda de apetite } \\
\hline Sim & 1,94 & $1,33-2,84$ & 0,001 & 1,92 & $1,33-2,79$ & 0,001 \\
\hline Não & 1,00 & & . & 1,00 & & \\
\hline \multicolumn{7}{|l|}{ Problema para mastigar ou deglutir } \\
\hline Sim & 2,13 & $1,28-3,57$ & 0,004 & 2,11 & $1,27-3,50$ & 0,004 \\
\hline Não & 1,00 & & . & 1,00 & & \\
\hline \multicolumn{7}{|l|}{ Condições de saúde bucal } \\
\hline \multicolumn{7}{|l|}{ Edentulismo } \\
\hline Sim & 0,60 & $0,42-0,84$ & 0,003 & 0,57 & $0,42-0,79$ & 0,001 \\
\hline Não & 1,00 & & & 1,00 & & \\
\hline \multicolumn{7}{|c|}{ Necessidade subjetiva de usar prótese } \\
\hline Sim & 5,08 & $3,58-7,21$ & $<0,001$ & 5,36 & $3,93-7,32$ & $<0,001$ \\
\hline Não & 1,00 & & & & & \\
\hline \multicolumn{7}{|c|}{ Uso e necessidade de prótese superior } \\
\hline Não usa e não necessita & 0,81 & $0,41-1,60$ & 0,542 & 0,78 & $0,41-1,48$ & 0,444 \\
\hline Não usa e necessita & 2,54 & $1,67-3,87$ & $<0,001$ & 1,94 & $1,27-2,96$ & 0,002 \\
\hline Usa e não necessita & 0,93 & $0,60-1,44$ & 0,754 & 0,77 & $0,47-1,26$ & 0,305 \\
\hline Usa e necessita & 1,00 & & & 1,00 & & \\
\hline
\end{tabular}

IC95\%: intervalo de 95\% de confiança; OR: odds ratio.

* Ajustado pela presença de depressão. 
Modelo de regressão ordinal final para a satisfação com a saúde bucal para o gênero feminino segundo características individuais, ambiente externo, disponibilidade de recursos, comportamentos de saúde, uso de serviços odontológicos e condições de saúde bucal. Sete Lagoas, Minas Gerais, Brasil, 2012.

\begin{tabular}{|c|c|c|c|c|c|c|}
\hline \multirow[t]{2}{*}{ Variáveis } & \multicolumn{3}{|c|}{ Modelo bruto } & \multicolumn{3}{|c|}{ Modelo ajustado * } \\
\hline & OR & IC95\% & Valor de $p$ & OR & IC95\% & Valor de $p$ \\
\hline \multicolumn{7}{|l|}{ Limiares do modelo ordinal } \\
\hline Insatisfeito & 3,81 & $1,19-12,16$ & 0,024 & 3,57 & $1,07-11,95$ & 0,039 \\
\hline Nem insatisfeito nem satisfeito & 1,70 & $0,54-5,42$ & 0,367 & 1,61 & $0,48-5,37$ & 0,438 \\
\hline \multicolumn{7}{|l|}{ Características individuais } \\
\hline \multicolumn{7}{|l|}{ Cor da pele auto relatada } \\
\hline Branca & 1,67 & $0,61-4,54$ & 0,318 & 1,28 & $0,64-2,56$ & 0,493 \\
\hline Preta & 2,06 & $0,74-5,78$ & 0,168 & 1,69 & $0,83-3,46$ & 0,151 \\
\hline Amarela & 1,70 & $0,63-4,60$ & 0,296 & 1,38 & $0,70-2,76$ & 0,354 \\
\hline Parda & 5,19 & $1,32-20,32$ & 0,018 & 1,91 & $0,69-5,31$ & 0,216 \\
\hline Indígena & 1,00 & & & 1,00 & & \\
\hline \multicolumn{7}{|l|}{ Ambiente externo } \\
\hline \multicolumn{7}{|l|}{ Tempo de moradia (anos) } \\
\hline$\leq 10$ & 1,34 & $0,95-1,90$ & 0,097 & 1,39 & $1,07-1,82$ & 0,015 \\
\hline $11-24$ & 1,79 & $1,27-2,52$ & 0,001 & 1,49 & $1,14-1,95$ & 0,003 \\
\hline $25-37$ & 1,66 & $1,19-2,34$ & 0,003 & 1,54 & $1,18-2,02$ & 0,002 \\
\hline 38 e mais & 1,00 & & & 1,00 & & \\
\hline \multicolumn{7}{|l|}{ Esgoto } \\
\hline Rede geral canalizada & 0,53 & $0,32-0,88$ & 0,014 & 0,58 & $0,40-0,83$ & 0,004 \\
\hline Fossa & 1,00 & & & 1,00 & & \\
\hline \multicolumn{7}{|l|}{ Comportamentos de saúde } \\
\hline \multicolumn{7}{|c|}{ Problema para mastigar ou deglutir } \\
\hline $\operatorname{Sim}$ & 2,02 & $1,46-2,82$ & $<0,001$ & 2,20 & $1,68-2,89$ & $<0,001$ \\
\hline Não & 1,00 & & & 1,00 & & \\
\hline \multicolumn{7}{|l|}{ Refeições por dia } \\
\hline 1 & 1,80 & $0,99-3,27$ & 0,056 & 0,72 & $0,44-1,15$ & 0,171 \\
\hline 2 & 0,91 & $0,65-1,28$ & 0,602 & 1,07 & $0,83-1,38$ & 0,612 \\
\hline 3 & 0,74 & $0,57-0,98$ & 0,034 & 1,23 & $0,99-1,52$ & 0,062 \\
\hline 4 ou mais & 1,00 & & & 1,00 & & \\
\hline \multicolumn{7}{|l|}{ Autoavaliação do peso } \\
\hline Abaixo do peso & 1,17 & $0,76-1,81$ & 0,479 & & & \\
\hline No peso normal & 0,77 & $0,59-1,00$ & 0,051 & & & \\
\hline Acima do peso & 1,00 & & & & & \\
\hline \multicolumn{7}{|l|}{ Uso de serviços odontológicos } \\
\hline \multicolumn{7}{|l|}{ Plano odontológico } \\
\hline $\operatorname{Sim}$ & 0,55 & $0,38-0,81$ & 0,003 & 1,40 & $1,03-1,91$ & 0,031 \\
\hline Não & 1,00 & & & 1,00 & & \\
\hline \multicolumn{7}{|l|}{ Condições de saúde bucal } \\
\hline \multicolumn{7}{|l|}{ Edentulismo } \\
\hline Sim & 0,48 & $0,37-0,62$ & $<0,001$ & 0,54 & $0,44-0,66$ & $<0,001$ \\
\hline Não & 1,00 & & & 1,00 & & \\
\hline \multicolumn{7}{|c|}{ Necessidade subjetiva de usar prótese } \\
\hline $\operatorname{Sim}$ & 4,66 & $3,64-5,98$ & $<0,001$ & 4,79 & $3,95-5,80$ & $<0,001$ \\
\hline Não & 1,00 & & & 1,00 & & \\
\hline
\end{tabular}

IC95\%: intervalo de 95\% de confiança; OR: odds ratio.

* Ajustado pela presença de depressão. 
precisa entre rede de esgoto e satisfação com a saúde bucal desses idosos.

No presente estudo, somente no modelo final ajustado para o gênero feminino o tempo de moradia se manteve associado ao desfecho. Um trabalho realizado em Campinas mostrou que o idoso que mora sozinho avalia sua saúde geral mais positivamente em relação aos que moram acompanhados 30 .

O perfil socioeconômico da grande maioria dos idosos brasileiros, em especial na zona rural, é marcado por condições de moradia e saneamento sanitário precárias, baixo nível de renda e de escolaridade, e atividade remunerada em idades avançadas 31,32 . Além disso, sabe-se que um maior nível de escolaridade e renda favorece maior busca por informações de saúde e uma autoavaliação mais positiva da saúde 16,28 . Nesse contexto, esperava-se que as variáveis de disponibilidade de recursos estivessem associadas ao desfecho no modelo final. Apenas na análise univariada houve associação significantemente inversa entre renda e satisfação com a saúde bucal para os homens.

Os aspectos mais importantes que compõem o conceito de percepção da saúde perpassam a compreensão dos comportamentos de saúde e qualidade de vida relacionada à saúde 12 . Apesar disso, as variáveis que dizem respeito aos comportamentos de saúde como tabagismo, alcoolismo, atividade física e sono, não contribuíram para explicar a variabilidade do grau de satisfação bucal entre os idosos do presente estudo. Na análise por nível, observou-se que os homens fumantes tinham 1,6 mais chance $(p=0,014)$ de estarem menos satisfeitos com a própria saúde bucal.

Os idosos que relataram problemas para mastigar os alimentos tiveram 2,11 e 2,02 mais chances de estar menos satisfeitos com a saúde bucal para os gêneros masculino e feminino, respectivamente. No modelo ajustado masculino, a perda de apetite nos últimos seis meses também se manteve associada à menor satisfação com a saúde bucal. Para o gênero feminino, o maior número de refeições foi fator preditor de maior satisfação com a saúde. Entretanto, quando a variável depressão foi inserida no modelo, essa associação não foi estatisticamente significante.

Segundo Lima et al. 33, os idosos se adaptam durante o processo de mastigação a fim de manter sua dieta o mais próximo possível da realizada quando jovem. Por outro lado, a perda dentária e o uso de próteses influenciam diretamente a capacidade mastigatória, a percepção do sabor e a textura dos alimentos, podendo resultar em menor ingestão de nutrientes, prejudicando o estado nutricional do idoso 34 .
Para o presente estudo as questões relacionadas à alimentação e à mastigação foram importantes na avaliação da satisfação com a saúde bucal. Em um trabalho realizado na Índia, cerca de $30 \%$ dos idosos acharam que não houve mudanças na dieta por causa da perda dentária 35 .

Outro achado interessante deste estudo refere-se à autoavaliação do peso corporal entre os idosos. Para ambos os gêneros, os idosos com peso normal avaliaram mais positivamente a sua saúde bucal em relação aos obesos. Entretanto, essa associação não foi suficientemente forte para ser mantida no modelo ajustado. A obesidade é um problema nutricional complexo que pode causar graves prejuízos à qualidade de vida $3 \mathrm{e}$ merece uma atenção melhor por parte do profissional de saúde e da equipe interdisciplinar para se obter ações coletivas eficientes.

O uso dos serviços odontológicos é um importante preditor para uma autoavaliação positiva da saúde bucal 16,29. Neste estudo, as variáveis sobre acesso e uso dos serviços odontológicos não estiveram associadas ao desfecho, com exceção do plano odontológico para o gênero feminino.

No último nível da análise aparecem as condições de saúde bucal, tanto as medidas objetivas (edentulismo, avaliação clínica do uso e necessidade de prótese) e a autoavaliação da necessidade de prótese. No estudo de Atchison et al. 28 , quanto maior o número de dentes permanentes presentes mais positivamente o idoso avalia a sua saúde bucal. Na presente pesquisa verificou-se que a satisfação com a saúde bucal esteve significativa e negativamente associada com o edentulismo, em todos os modelos da análise. No modelo ajustado, os idosos edêntulos dos gêneros masculino e feminino tinham $57 \%$ e $56 \%$, respectivamente, mais chances de estarem satisfeitos quando comparados aos não edêntulos. Enquanto $47,7 \%$ dos idosos insatisfeitos não usavam nenhuma prótese e tinham necessidade, $39,7 \%$ dos satisfeitos usavam prótese e não necessitavam de substituição.

Em três estudos brasileiros 6,10,29 os idosos desdentados avaliaram positivamente a saúde bucal, possivelmente por se verem livres de um ciclo de péssimas condições bucais (dentes cariados, dor de dente). Alguns trabalhos tentaram entender essa inversão de gradiente de associação de diversas maneiras, sendo que a maioria dos autores relata que essa associação é fraca 9,10,12,15. Outra possível explicação seria assumir que diferentes contextos podem influenciar a direção de associação entre percepção da saúde bucal e condições objetivas de saúde. Entretanto, falta maior poder de comparabilidade entre os estudos nacionais em relação ao grupo de pes- 
quisadores que trabalha esse tema há muitos anos.

A avaliação da necessidade de prótese é um assunto controverso, pois os julgamentos do profissional e do idoso são, muitas vezes, completamente divergentes. Os idosos que necessitavam usar/trocar prótese estavam menos satisfeitos com a própria saúde bucal. Apesar disso, a maioria dos idosos desta investigação que não usava nenhum tipo de prótese e necessitava não percebeu a necessidade de usá-la. Ariga et al. 35 mostraram que dos 70,3\% dos idosos que tinham necessidade de usar prótese apenas $14,4 \%$ perceberam a necessidade de substituir dentes perdidos. Percebe-se que os idosos têm percepções diferentes relacionadas à condição de saúde bucal e à necessidade de tratamento odontológico 4 .

Por último, destaca-se que a avaliação clínica do uso e necessidade de prótese em idosos é diferente entre as arcadas superior e inferior, especialmente em relação ao uso de prótese total 36. Neste estudo, somente um pequeno porcentual dos idosos não necessitava nem usava qualquer tipo de prótese inferior $(12,6 \%)$ ou superior $(8,1 \%)$.

Considerando-se o impacto da saúde bucal na saúde geral e no bem-estar do idoso, outro objetivo da presente investigação foi avaliar a prevalência de edentulismo em relação ao gênero e faixa etária. A perda dentária não pode ser mais considerada uma consequência normal do envelhecimento. Trata-se de um processo cumulativo de exposição à cárie, doença periodontal, periapical ou trauma, que acarreta problemas na mastigação, satisfação com a aparência dos dentes, autopercepção de saúde ruim e necessidade de reabilitação protética 5,15 .

A prevalência de edentulismo deste estudo foi elevada $(66,4 \%)$, sendo maior ainda entre as mulheres $(\mathrm{p}=0,004)$, resultado semelhante a outras investigações 14,23. De acordo com a literatura, com o aumento da idade tem-se a redução do número de dentes, aumento do índice CPO e do percentual de dentes perdidos por cárie 37 . Neste estudo, a razão de prevalência de edentulismo foi estatisticamente maior apenas para as idosas mais jovens, revelando muito mais uma particularidade do gênero feminino do que diferenças reais entre as cinco faixas etárias do envelhecimento. Esses resultados refletem a situação nacional 5, mas devem ser interpretados com cautela, uma vez que a maioria dos idosos deste e de diversos estudos é de mulheres. O tamanho, as estratificações e correções da amostra minimizam esses efeitos, possibilitando as comparações realizadas neste trabalho.

Em relação à utilização do modelo multidimensional por meio da regressão logística ordi- nal pode-se afirmar que foi possível identificar os fatores determinantes para satisfação com a saúde bucal, seguindo os níveis hierárquicos teóricos de determinação de maneira independente.

Para a variável dependente que apresenta três categorias de respostas, o modelo apresenta dois limiares (thresholds). É interessante observar que o ponto de corte entre a categoria do meio (nem insatisfeito nem satisfeito) e a superior (satisfeito) para o modelo feminino não foi significante ( $\mathrm{p}=0,367$ ), mesmo após o ajuste do modelo ( $\mathrm{p}=$ 0,438 ). Possivelmente isso indica que as idosas desses dois grupos podem ter percepções semelhantes de respostas em relação às variáveis independentes do modelo, mas não há uma base teórica concreta para garantir esta interpretação.

A taxa de resposta do presente trabalho pode ser considerada alta $(98,8 \%)$, sendo este um dos poucos estudos domiciliares com idosos que obteve amostra probabilística suficiente para realizar aferições e comparações por gênero. De modo geral, o perfil epidemiológico da saúde bucal de idosos no Brasil tem sido avaliado por meio de pesquisas com amostras não probabilísticas, tais como usuários de centros de saúde e centros de convivência ${ }^{2}$, idosos institucionalizados 17 e usando resultados referentes aos inquéritos epidemiológicos do Ministério da Saúde 11. Entretanto, faz-se necessário especificar que os resultados encontrados são válidos e representativos para a população do município que vive em comunidade, sem déficits cognitivos e/ou físicos importantes.

Finalmente, é importante discutir algumas limitações do trabalho. Este estudo, em que a maioria dos idosos era composta por edêntulos, pode ter tido influência nos resultados encontrados quando comparado à satisfação com a saúde bucal em outra faixa etária. Esses resultados fazem parte da linha base do projeto sobre envelhecimento ativo. Por isso, ainda não é possível determinar se existe uma relação temporal entre a satisfação com a saúde bucal e as demais variáveis analisadas. Além disso, a incorporação de outras variáveis e o geoprocessamento dos dados poderão ajudar a ampliar a discussão levantada neste trabalho.

Destaca-se ainda que a medida da satisfação com a saúde bucal é influenciada diretamente pela memória do entrevistado, pelas suas características físicas e psicológicas, e também pelos aspectos contextuais e culturais de cada grupo populacional. Portanto, quantificar o que é subjetivo é sempre limitado, pois envolve valores e sentimentos que podem mudar ao longo da vida e até mesmo em um curto espaço de tempo 10.

Em suma, o processo que relaciona a autopercepção às variáveis objetivas e subjetivas de 
saúde é dinâmico. Sendo assim, as ações integradas de promoção da saúde e do bem-estar que abordam, simultaneamente, os principais fatores determinantes da autopercepção da saúde poderão contribuir significativamente para a qualidade de vida dos idosos 8,23.

\section{Conclusão}

Conclui-se que a satisfação com a saúde bucal dos idosos deste estudo é diferente entre os gêneros e influenciada fortemente pelas condições de saúde bucal e comportamentos de saúde.

\section{Resumen}

Los objetivos de este estudio fueron identificar las diferencias de género, en relación con factores asociados a la satisfacción con la salud bucal de ancianos, con el fin de estimar la prevalencia de edentulismo por género y edad. Se incluyó a 2.052 personas mayores de 60 años y más que residen en Sete Lagoas, Minas Gerais, Brasil. La regresión de Poisson se utilizó para estimar la prevalencia de edentulismo. La asociación entre la satisfacción bucal y las variables independientes se realizó mediante el modelo de regresión ordinal multidimensional jerárquico. La prevalencia de edentulismo en hombres y mujeres era de un $63 \%$ y un $68,8 \%$, respectivamente. La mayoría de los hombres de edad avanzada (63\%) y mujeres (57\%) estaban satisfechos con su salud bucal. El modelo ajustado final, para ambos sexos, fue desarrollado por variables relacionadas con el ambiente externo, las conductas de salud y las condiciones de salud bucal. Se concluye que la satisfacción con la salud bucal está fuertemente influenciada por el estado de salud oral y las conductas de salud, asimismo, los factores asociados son diferentes para cada género.

Salud Bucal; Anciano; Autoimagen
Para o gênero feminino, a prevalência de edentulismo foi maior nas faixas etárias inferiores a 70 anos. Entre os homens, não observouse diferenças significantes entre as prevalências por faixa etária. Quando comparou-se homens e mulheres, verificamos uma razão de prevalência maior na faixa etária entre 65 e 69 anos.

As discussões de gênero no envelhecimento apresentadas neste estudo precisam ser aprofundadas em relação às condições e percepção da saúde bucal, pois existem particularidades de cada grupo que podem passar despercebidas nas análises rotineiras.

\section{Colaboradores}

A. C. V. Campos realizou a coleta dos dados, análise estatística e redação do artigo. A. M. D. Vargas realizou a revisão crítica e intelectual do conteúdo. E. F. Ferreira participou da concepção do projeto e interpretação dos dados. Todos os autores aprovaram a versão final do artigo.

\section{Agradecimentos}

A. C. V. Campos recebeu auxílio para a realização da pesquisa mediante bolsa concedida pelo CNPq, na modalidade doutorado no país (no 141307/2011-0) e o projeto foi financiado pelo CNPq (no 14/2011, protocolo 1829669704655455). 


\section{Referências}

1. Ministério da Saúde. SBBrasil 2010. Pesquisa Nacional de Saúde Bucal. Projeto técnico. http:// dab.saude.gov.br/CNSB/sbbrasil/download.htm (acessado em 24/Abr/2011).

2. Moreira RS, Nico LS, Tomita NE, Ruiz T. A saúde bucal do idoso brasileiro: revisão sistemática sobre o quadro epidemiológico e acesso aos serviços de saúde bucal. Cad Saúde Pública 2005; 21:1665-75.

3. Costa EHM, Saintrain MVL, Vieira APGF. Autopercepção da condição de saúde bucal em idosos institucionalizados e não institucionalizados. Ciênc Saúde Coletiva 2010; 15:2925-30.

4. Martins AMEBL, Barreto SM, Pordeus IA. Fatores relacionados à autopercepção da necessidade de tratamento odontológico entre idosos. Rev Saúde Pública 2008; 42:487-96.

5. Silva DD, Sousa MLR, Wada RS. Autopercepção e condições de saúde bucal em uma população de idosos. Cad Saúde Pública 2005; 21:1251-9.

6. Haikal DS, Paula AMB, Martins AMEBL, Moreira AN, Ferreira EF. Autopercepção da saúde bucal e impacto na qualidade de vida do idoso: uma abordagem quanti-qualitativa. Ciênc Saúde Coletiva 2011; 16:3317-29.

7. Andersen RM, Davidson PL. Ethnicity, aging, and oral health outcomes: a conceptual framework. Adv Dent Res 1997; 11:203-9.

8. Locker D, Gibson B. Discrepancies between selfratings of and satisfaction with oral health in two older adult populations. Community Dent Oral Epidemiol 2005; 33:280-8.

9. Locker D, Clarke M, Payne B. Self perceived oral health status, psychological well-being, and life satisfaction in an older population. J Dent Res 2000; 79:970-5.

10. Martins AMEBL, Barreto SM, Pordeus IA. Auto-avaliação de saúde bucal em idosos: análise com base em modelo multidimensional. Cad Saúde Pública 2009; 25:421-35.

11. Matos DL, Lima-Costa MF. Auto-avaliação da saúde bucal entre adultos e idosos residentes na Região Sudeste: resultados do Projeto SB-Brasil, 2003. Cad Saúde Pública 2006; 22:1699-707.

12. Silva SRC, Fernandes RAC. Autopercepção das condições de saúde bucal por idosos. Rev Saúde Pública 2001; 35:349-55.

13. Paskulin LMG, Aires M, Gonçales AV, Kottwitz CCB, Morais EP, Brondani MA. Aging, diversity, and health: the Brazilian and the Canadian context. Acta Paul Enferm 2011; 24:851-6.

14. Cardoso EM, Parente RCP, Vettore MV, Rebelo MAB. Condição de saúde bucal em idosos residentes no Município de Manaus, Amazonas: estimativas por sexo. Rev Bras Epidemiol 2011; 14:131-40.

15. Moreira RS, Nico LS, Tomita NE. Oral health conditions among the elderly in southeastern São Paulo state. J Appl Oral Sci 2009; 17:170-8.

16. Mendonça HLC, Szwarcwald CL, Damacena GN. Autoavaliação de saúde bucal: resultados da Pesquisa Mundial de Saúde - atenção básica em quatro municípios do Estado do Rio de Janeiro, Brasil, 2005. Cad Saúde Pública 2012; 28:1927-38.
17. Carneiro RMV, Silva DD, Souza MLR, Wada RS Saúde bucal de idosos institucionalizados, zona leste de São Paulo, Brasil, 1999. Cad Saúde Pública 2005; 21:1709-16.

18. Instituto Brasileiro de Geografia e Estatística. Contagem da população 2009. http://www.ibge.gov.br. (acessado em 07/Abr/2011).

19. Sete Lagoas. Lei no 7.804 de 14 de outubro de 2009 de Sete Lagoas. Define as Regiões Administrativas - RA no perímetro urbano do distrito sede do $\mathrm{Mu}$ nicípio de Sete Lagoas. http://www.jusbrasil.com. br/legislacao/834407/lei-7804-09-sete-lagoas. (acessado em 7/Abr/2011).

20. Santos JLF, Lebrão ML, Duarte YAO, Lima FD. Functional performance of the elderly in instrumental activities of daily living: an analysis in the municipality of São Paulo, Brazil. Cad Saúde Pública 2008; 24:879-86.

21. World Health Organization. Oral health surveys: basic methods. 4th Ed. http://www2.paho.org/hq/ dmdocuments/2009/OH_st_Esurv.pdf. (acessado em 07/Abr/2011).

22. Andersen RM, Davidson PL. Ethnicity, aging, and oral health outcomes: a conceptual framework. Adv Dent Res 1997; 11:203-9.

23. Martins AB, Dos Santos CM, Hilgert JB, de Marchi RJ, Hugo FN, Pereira Padilha DM. Resilience and self-perceived oral health: a hierarchical approach. J Am Geriatr Soc 2011; 59:725-31.

24. De Carlo LT. Using the PLUM procedure of SPSS to fit unequal variance and generalized signal detection models. Behavior Research Methods, Instruments, \& Computers 2003; 35:49-56.

25. Blazer DG. How do you feel about...? Health outcomes in late life and self-perceptions of health and well-being. Gerontologist 2008; 48:415-22.

26. Almeida OP, Almeida SA. Confiabilidade da versão brasileira da Escala de Depressão em Geriatria (GDS) versão reduzida. Arq Neuropsiquiatr 1999; 57:421-6.

27. McCullagh P. Rgression models for ordinal data. J R Stat Soc Series B Stat Methodol 1980; 42:109-42.

28. Atchison KA, Matthias RE, Dolan TA, Lubben JE, De Jong F, Mayer-Oakes SA. Comparison of oral health ratying by dentists and dentate elders. J Public Health Dent 1993; 53:223-30.

29. Bulgarelli AF, Manço ARX. Idosos vivendo na comunidade e a satisfação com a própria saúde bucal. Ciênc Saúde Coletiva 2008; 13:1165-74.

30. Borim FSA, Barros MBA, Neri AL. Autoavaliação da saúde em idosos: pesquisa de base populacional no Município de Campinas, São Paulo, Brasil. Cad Saúde Pública 2012; 28:769-80.

31. Anderson MIP. Saúde e condições de vida do idoso no Brasil. Textos Envelhecimento 1998; 1:7-22.

32. Perelman J, Fernandes A, Mateus C. Gender disparities in health and healthcare: results from the Portuguese National Health Interview Survey. Cad Saúde Pública 2012; 28:2339-48. 
33. Lima RMF, Amaral AKFJ, Aroucha EBL, Vasconcelos TMJ, Silva HJ, Cunha DA. Adaptações na mastigação, deglutição e fonoarticulação em idosos de instituição de longa permanência. Rev CEFAC 2009; 11 Suppl 3:405-22.

34. Sheiham A, Steele JG, Marcenes W, Lowe C, Finch S, Bates CJ, et al. The relationship among dental status, nutrient intake, and nutritional status in older people J Dent Res 2001; 80:408-13.

35. Ariga P, Bridgitte A, Rangarajan V, Philip JM. Edentulousness, denture wear and denture needs of the elderly in rural South India. Iran J Public Health 2012; 41:40-3.
36. Colussi CF, Freitas SFT. Aspectos epidemiológicos da saúde bucal do idoso no Brasil. Cad Saúde Pública 2002; 18:1313-20.

37. Silveira EA, Kac G, Barbosa LS. Prevalência e fatores associados à obesidade em idosos residentes em Pelotas, Rio Grande do Sul, Brasil: classificação da obesidade segundo dois pontos de corte do índice de massa corporal. Cad Saúde Pública 2009 25:1569-77.

Recebido em 18/Abr/2013

Versão final reapresentada em 27/Ago/2013

Aprovado em 04/Out/2013 